Supporting Information for ES\&T manuscript es052067y:

\title{
Perfluorooctanesulfonate and Related Fluorochemicals in Human Blood Samples from China
}

Authors: LEO, W.Y. YEUNG, M.K. SO, GUI-BIN JIANG, S. TANIYASU, N. YAMASHITA, MAOYONG SONG, YONGNING WU, JINGGUANG LI, J.P. GIESY, K.S. GURUGE AND PAUL K. S. LAM

(Pages 3, Table) 
Table showing mean, standard deviation, median, minimum, and maximum perfluorochemical concentrations $(\mathrm{ng} / \mathrm{mL})$ in whole human blood from different cities in China stratified by donor gender

\begin{tabular}{|c|c|c|c|c|c|c|c|c|c|}
\hline \multicolumn{2}{|c|}{ Gender (no. of samples) } & PFOS & PFHxS & PFOSA & PFUnDA & PFDA & PFNA & PFOA & Total PFC \\
\hline \multicolumn{10}{|c|}{ Beijing (Hebei) } \\
\hline \multirow[t]{5}{*}{ Male $(n=5)$} & mean & 42.9 & 1.89 & 1.47 & 0.28 & 0.17 & 0.46 & 0.61 & 47.8 \\
\hline & S.D. & 17.4 & 0.91 & 0.58 & 0.12 & 0.06 & 0.1 & 0.12 & 18.4 \\
\hline & median & 40.4 & 1.75 & 1.47 & 0.27 & 0.17 & 0.46 & 0.61 & 45.5 \\
\hline & $\min$ & 16.6 & 0.72 & 0.73 & 0.15 & 0.1 & 0.32 & 0.43 & 19.8 \\
\hline & $\max$ & 63.9 & 3.39 & 2.17 & 0.47 & 0.25 & 0.61 & 0.76 & 70.5 \\
\hline \multirow[t]{6}{*}{ Female $(\mathrm{n}=5)$} & mean & 25.9 & 1.1 & 1.1 & 0.22 & 0.14 & 0.31 & 0.85 & 29.6 \\
\hline & S.D. & 13.7 & 0.6 & 0.9 & 0.04 & 0.03 & 0.04 & 0.81 & 14.8 \\
\hline & median & 21.7 & 1.02 & 0.79 & 0.23 & 0.13 & 0.29 & 0.53 & 25.2 \\
\hline & $\min$ & 12.1 & 0.39 & 0.26 & 0.15 & 0.11 & 0.26 & 0.3 & 13.6 \\
\hline & $\max$ & 52.8 & 2.31 & 2.81 & 0.3 & 0.2 & 0.37 & 2.4 & 58.1 \\
\hline & \multicolumn{9}{|c|}{ Fuzhou (Fujian) } \\
\hline \multirow[t]{5}{*}{ Male $(n=5)$} & mean & 29.8 & 1.48 & 0.54 & 0.56 & 0.26 & 0.58 & 1.2 & 34.4 \\
\hline & S.D. & 13.9 & 0.68 & 0.1 & 0.23 & 0.06 & 0.2 & 0.57 & 14 \\
\hline & median & 20.5 & 0.75 & 0.44 & 0.62 & 0.29 & 0.55 & 0.91 & 34.2 \\
\hline & $\min$ & 28.9 & 1.44 & 0.53 & 0.47 & 0.25 & 0.53 & 0.88 & 16.7 \\
\hline & $\max$ & 56.8 & 2.79 & 0.68 & 1.06 & 0.38 & 0.98 & 2.09 & 61.7 \\
\hline \multirow[t]{6}{*}{ Female $(n=5)$} & mean & 17.1 & 0.46 & 0.37 & 0.77 & 0.33 & 0.59 & 0.9 & 20.5 \\
\hline & S.D. & 8.15 & 0.16 & 0.05 & 0.25 & 0.08 & 0.14 & 0.21 & 8.3 \\
\hline & median & 15.4 & 0.44 & 0.36 & 0.82 & 0.31 & 0.57 & 0.95 & 17.8 \\
\hline & $\min$ & 8.83 & 0.26 & 0.31 & 0.41 & 0.23 & 0.45 & 0.55 & 12.2 \\
\hline & $\max$ & 32.3 & 0.68 & 0.49 & 1.14 & 0.47 & 0.87 & 1.2 & 36.1 \\
\hline & \multicolumn{9}{|c|}{ Xiamen (Fujian) } \\
\hline \multirow[t]{5}{*}{ Male $(n=4)$} & mean & 30.2 & 1.05 & 1.68 & $0.67^{\circ}$ & 0.44 & 0.66 & 1.39 & 36 \\
\hline & S.D. & 19.9 & 0.58 & 0.3 & 0.6 & 0.47 & 0.57 & 1.23 & 23.2 \\
\hline & median & 20.1 & 0.81 & 1.57 & 0.45 & 0.24 & 0.44 & 0.99 & 24.5 \\
\hline & $\min$ & 18.3 & 0.6 & 1.25 & 0.18 & 0.12 & 0.21 & 0.25 & 21.9 \\
\hline & $\max$ & 69.9 & 2.21 & 2.28 & 1.8 & 1.36 & 1.55 & 3.49 & 82.1 \\
\hline \multirow[t]{6}{*}{ Female $(n=3)$} & mean & 16.6 & 0.67 & 1.05 & 0.73 & 0.4 & 0.46 & 0.62 & 20.5 \\
\hline & S.D. & 11.8 & 0.6 & 0.76 & 0.27 & 0.09 & 0.15 & 0.29 & 13.8 \\
\hline & median & 10.2 & 0.33 & 0.64 & 0.78 & 0.43 & 0.64 & 0.66 & 13.6 \\
\hline & $\min$ & 6.97 & 0.22 & 0.45 & 0.39 & 0.28 & 0.25 & 0.27 & 8.84 \\
\hline & $\max$ & 33.8 & 1.5 & 2.16 & 1.08 & 0.49 & 0.51 & 0.97 & 40.5 \\
\hline & \multicolumn{9}{|c|}{ Guiyang (Guizhou) } \\
\hline \multirow[t]{5}{*}{ Male $(n=6)$} & mean & 22.4 & 1.07 & 1.26 & 0.15 & 0.11 & 0.26 & 0.31 & 25.5 \\
\hline & S.D. & 16.4 & 0.98 & 0.96 & 0.04 & 0 & 0.09 & 0.07 & 18.4 \\
\hline & median & 17 & 0.83 & 0.83 & 0.14 & 0.11 & 0.25 & 0.31 & 18.8 \\
\hline & Min & 4.65 & 0.21 & 0.68 & $<0.1$ & $<0.1$ & 0.15 & 0.21 & 6.26 \\
\hline & Max & 58.2 & 3.2 & 3.46 & 0.22 & 0.11 & 0.44 & 0.44 & 66 \\
\hline \multirow[t]{5}{*}{ Female $(n=5)$} & Mean & 26.3 & 1.1 & 0.84 & 0.13 & 0.08 & 0.28 & 0.31 & 29 \\
\hline & S.D. & 13.4 & 0.7 & 0.87 & 0.08 & 0.07 & 0.1 & 0.08 & 14.4 \\
\hline & median & 21.9 & 0.89 & 0.47 & 0.14 & 0.1 & 0.24 & 0.33 & 23.9 \\
\hline & $\min$ & 10.1 & 0.29 & 0.34 & $<0.1$ & $<0.1$ & 0.15 & 0.15 & 11.1 \\
\hline & $\max$ & 48.9 & 2.16 & 2.56 & 0.23 & 0.16 & 0.4 & 0.39 & 52.1 \\
\hline
\end{tabular}




\begin{tabular}{|c|c|c|c|c|c|c|c|c|c|}
\hline \multirow[b]{2}{*}{ Male $(n=5)$} & \multicolumn{9}{|c|}{ Zhengzhou (Henan) } \\
\hline & mean & 19.3 & 0.9 & 1.06 & 0.14 & 0.1 & 0.21 & 0.32 & 21.9 \\
\hline & S.D. & 12.5 & 0.67 & 0.82 & 0.03 & 0.01 & 0.06 & 0.09 & 13.5 \\
\hline & median & 15.7 & 0.57 & 0.7 & 0.15 & 0.1 & 0.22 & 0.33 & 18.9 \\
\hline & $\min$ & 7.25 & 0.31 & 0.28 & $<0.1$ & $<0.1$ & $<0.1$ & 0.18 & 8.22 \\
\hline & $\max$ & 45.7 & 2.11 & 2.14 & 0.18 & 0.11 & 0.28 & 0.47 & 49.8 \\
\hline \multirow[t]{6}{*}{ Female $(\mathrm{n}=5)$} & Mean & 11.3 & 0.44 & 0.52 & 0.16 & 0.12 & 0.2 & 0.2 & 12.8 \\
\hline & S.D. & 2.57 & 0.14 & 0.26 & 0.06 & 0.02 & 0.08 & 0.05 & 2.99 \\
\hline & median & 12.2 & 0.38 & 0.36 & 0.15 & 0.11 & 0.18 & 0.19 & 13.9 \\
\hline & $\min$ & 7.14 & 0.3 & 0.3 & $<0.1$ & $<0.1$ & 0.12 & 0.13 & 8.15 \\
\hline & $\max$ & 14.3 & 0.66 & 0.98 & 0.25 & 0.16 & 0.36 & 0.3 & 16.5 \\
\hline & \multicolumn{9}{|c|}{ Jintan (Jiangsu) } \\
\hline \multirow[t]{5}{*}{ Male $(n=5)$} & mean & 4.7 & 0.19 & 0.41 & 1.18 & 1.35 & 1.03 & 1.55 & 10.4 \\
\hline & S.D. & 1.55 & 0.05 & 0.31 & 0.67 & 0.73 & 0.51 & 0.73 & 3.79 \\
\hline & median & 4.75 & 0.17 & 0.25 & 1.07 & 1.32 & 0.82 & 1.24 & 10 \\
\hline & $\min$ & 2.59 & 0.13 & 0.17 & 0.49 & 0.55 & 0.56 & 0.83 & 5.77 \\
\hline & $\max$ & 7.22 & 0.32 & 1.02 & 2.85 & 3.21 & 2.19 & 3.13 & 18.8 \\
\hline \multirow[t]{6}{*}{ Female $(n=5)$} & mean & 2.74 & 0.14 & 0.24 & 0.73 & 0.82 & 0.6 & 0.84 & 6.05 \\
\hline & S.D. & 0.69 & 0.03 & 0.16 & 0.36 & 0.4 & 0.29 & 0.44 & 1.94 \\
\hline & median & 2.58 & 0.15 & 0.13 & 0.57 & 0.66 & 0.44 & 0.76 & 5.5 \\
\hline & $\min$ & 1.69 & $<0.1$ & 0.11 & 0.46 & 0.53 & 0.36 & 0.38 & 4.02 \\
\hline & $\max$ & 3.87 & 0.17 & 0.51 & 1.43 & 1.61 & 1.1 & 1.56 & 9.59 \\
\hline & \multicolumn{9}{|c|}{ Shenyang (Liaoning) } \\
\hline \multirow[t]{5}{*}{ Male $(n=6)$} & mean & 71 & 2.44 & 1.95 & 0.17 & 0.12 & 0.39 & 0.63 & 76.7 \\
\hline & S.D. & 29.8 & 1.65 & 1.16 & 0.03 & 0.01 & 0.06 & 0.29 & 32.2 \\
\hline & median & 70.2 & 1.88 & 1.84 & 0.17 & 0.12 & 0.4 & 0.49 & 75.1 \\
\hline & $\min$ & 15.8 & 0.56 & 0.53 & 0.14 & 0.11 & 0.3 & 0.32 & 17.9 \\
\hline & $\max$ & 112 & 5.57 & 4.22 & 0.23 & 0.14 & 0.49 & 1.2 & 122 \\
\hline \multirow[t]{6}{*}{ Female $(n=4)$} & mean & 85.1 & 1.37 & 1.56 & 0.2 & 0.1 & 0.34 & 0.39 & 88.8 \\
\hline & S.D. & 44.6 & 0.69 & 0.98 & 0.04 & 0 & 0.13 & 0.14 & 46 \\
\hline & median & 69.5 & 1.22 & 1.43 & 0.19 & 0.1 & 0.29 & 0.36 & 72.9 \\
\hline & $\min$ & 38.7 & 0.71 & 0.44 & 0.14 & $<0.1$ & 0.23 & 0.25 & 40.5 \\
\hline & $\max$ & 155 & 2.87 & 3.07 & 0.25 & 0.1 & 0.57 & 0.59 & 162 \\
\hline & \multicolumn{9}{|c|}{ Wuhan (Hubei) } \\
\hline \multirow[t]{5}{*}{ Male $(n=6)$} & mean & 20.5 & 0.81 & 0.69 & 0.28 & 0.18 & 0.37 & 0.53 & 23.3 \\
\hline & S.D. & 9.29 & 0.41 & 0.23 & 0.12 & 0.07 & 0.15 & 0.24 & 10.2 \\
\hline & median & 21.2 & 0.7 & 0.67 & 0.27 & 0.17 & 0.34 & 0.49 & 24.2 \\
\hline & $\min$ & 9.69 & 0.31 & 0.41 & 0.12 & 0.07 & 0.16 & 0.19 & 11.2 \\
\hline & $\max$ & 35.1 & 1.4 & 1.02 & 0.54 & 0.32 & 0.67 & 0.92 & 39.2 \\
\hline \multirow[t]{2}{*}{ Female $(\mathrm{n}=1)$} & & 22.4 & 0.85 & 0.72 & 0.33 & 0.2 & 0.41 & 0.55 & 27.6 \\
\hline & \multicolumn{9}{|c|}{ Zhoushan (Zhejiang) } \\
\hline \multirow[t]{5}{*}{ Female $(n=10)$} & mean & 12 & 0.37 & 0.47 & 1.49 & 0.49 & 1.27 & 1.55 & 17.6 \\
\hline & S.D. & 6.46 & 0.21 & 0.21 & 0.43 & 0.16 & 0.45 & 0.85 & 8.07 \\
\hline & median & 10.1 & 0.27 & 0.41 & 1.36 & 0.42 & 1.19 & 1.3 & 16 \\
\hline & $\min$ & 5.38 & 0.13 & 0.25 & 0.9 & 0.34 & 0.72 & 0.57 & 8.8 \\
\hline & $\max$ & 25.6 & 0.91 & 0.86 & 2.58 & 0.87 & 2.27 & 3.26 & 32.6 \\
\hline
\end{tabular}

Samples with values below the limits of quantification were treated as zero in the calculation of mean and median. 